\title{
Erroneous Examples as Desirable Difficulty
}

\author{
Deanne Adams ${ }^{1}$, Bruce M. McLaren ${ }^{2}$, Richard E. Mayer ${ }^{1}$, George Goguadze $^{3}$, Seiji \\ Isotani $^{4}$ \\ ${ }^{1}$ University of California, Santa Barbara, U.S.A. \\ ${ }^{2}$ Carnegie Mellon University, U.S.A. \\ ${ }^{3}$ Leuphana University Lüneburg, Germany \\ ${ }^{4}$ The University of São Paulo, Brazil \\ bmclaren@cs.cmu.edu
}

\begin{abstract}
Erroneous examples, an unusual and challenging form of learning material, are arguably a type of desirable difficulty for students that could lead to deeper learning. In a series of studies we have done over the past three years involving web-based math instruction, the learning benefits of erroneous examples we have observed occured on delayed tests, as occurs in the desirable difficulties literature. This short paper briefly reviews the literature, summarizes our results, and speculates on how an adaptive version of our materials could better leverage desirable difficulties theory and lead to deeper student learning.
\end{abstract}

Keywords: erroneous examples, interactive problem solving, adaptation of problems, self-explanation, decimals, mathematics education

\section{Introduction}

Erroneous examples are step-by-step descriptions of how to solve a problem in which one or more of the steps are incorrect. In the studies we have done with erroneous examples over the past three years, focused on learning decimals using web-based, interactive materials, middle school students are prompted to find, explain, and fix error(s) in order to more deeply learn how to solve decimal problems.

Presenting students with challenge is central to the notion of learning with erroneous examples. Research on desirable difficulties has shown that it is possible to achieve long-term benefits if lessons are designed (or altered) to make them more challenging during learning $[1,2]$. Examples of desirable difficulties include mixing the order of tasks for practice (rather than providing tasks in blocked fashion); varying the frequency and timing of feedback (rather than providing immediate feedback); and varying tasks with a focus on generalizability. These changes to standard instructional practice have been shown to slow the rate of improvement in students' understanding during the learning process but lead to long-term benefits [1, 2].

The erroneous examples we work with can be viewed as presenting desirable difficulties for students in two ways. First, they are an unusual and challenging form of problem, in which students must find, explain, and correct errors, as opposed to the more standard practice of simply solving problems. Although this characteristic is not 
cited in the original definition of desirable difficulties [1], this type of challenge, which we believe promotes deeper cognitive processing, is also arguably a form of desirable difficulty. Second, the erroneous examples intervention of the present study provides the third type of challenge from Schmidt and Bjork's original desirable difficulties - varying of tasks - by prompting students to grapple with both erroneous examples and problems to solve in the intervention.

The domain we have focused on is decimals. A variety of studies have shown that students often have difficulty mastering decimals and have common and persistent misconceptions [3, 4], as well as problems that extend even into adulthood [5]. For instance, students often treat decimals as if they are whole numbers (e.g. they think 0.15 is greater than 0.8 , since 15 is greater than 8 , i.e., longer decimals are larger) or they think that all decimals are less than zero.

\section{Research on Erroneous Examples}

Research on erroneous examples derives from work on correct worked examples, which has attracted much attention in the literature and in empirical studies, e.g., [6]. The idea behind worked examples is that they free working memory, which has a limited capacity, which can be used to support learning of new knowledge. Erroneous examples may tax working memory somewhat during learning, but they also may engage students in a different form of active learning, particularly when coupled with self-explanation [7]. Erroneous examples may help students become better at evaluating and justifying solution procedures, which may, in turn, help them learn material at a deeper level. Empirical research in erroneous examples is nascent, but with encouraging results. For instance, Siegler [8] found that self-explaining both correct and incorrect examples (of mathematical equality) is more beneficial than selfexplaining correct examples only. Grosse and Renkl [9] studied whether explaining both correct and incorrect examples can help university students learn statistics. Their studies also showed learning benefits of erroneous examples but the benefit was only for learners with higher prior knowledge and for far transfer learning only. When errors were highlighted, on the other hand, low prior knowledge individuals did significantly better, while high prior knowledge students did not benefit, presumably because they were already able to identify the error on their own.

\section{Our Erroneous Examples Studies and Results}

Providing students with interactive erroneous examples is the approach that we take in our research. By interactive we mean that students are prompted to actively engage with the examples. More specifically, our computer-based materials first prompt a student to review an error made by a fictitious peer, next request that the student explain the error (from a multiple-choice list), then correct the error and explain how to solve problems of this type (again from a multiple-choice list). At every step the student's action is evaluated for correctness. 
We have conducted two previously published studies with interactive erroneous examples $[10,11]$. In the first study [10] an interactive erroneous examples condition did not lead to learning benefits compared to a worked examples condition and problem solving condition. We attributed this result to two things: (1) A cognitively taxing self-explanation step, in which students were prompted to complete explanations of incorrect steps by filling in two phrases of a sentence, using pulldown menus. Students struggled with this task, possibly undercutting the intended benefit we intended. (2) We did not prompt students to correct the errors and produce the correct answers themselves, a step we now believe to be a critical component of interactive erroneous examples.

Our second study [11] was conducted after revising the interactive erroneous examples along these two dimensions (i.e., simplifying the self-explanation step by prompting for only a single sentence completion phrase and prompting students to correct errors). With $100+$ students in each of two conditions - interactive erroneous examples and supported problem solving - an effect was found: students who worked with the interactive erroneous examples did significantly better than the problem solving students on a delayed posttest (but not on an immediate posttest).

Our third study, which will be published in a forthcoming journal article, employed the same materials as described in [11] but entailed a much larger population of students. More specifically, our latest results are a combination of the [11] results and the running of the study five more times at three additional schools over the course of a year. The total number of subjects per condition is more than three times that of [11] - over 300 students per condition. In addition, a third condition of 82 students, in which subjects were presented with erroneous examples in adaptive fashion, based on a Bayes Net assessment of their misconceptions on the pretest, was included in the final three versions of the study. These results indicate, once again, that students who worked with the interactive erroneous examples did significantly better than the problem solving students on a delayed posttest (but not on an immediate posttest). Surprisingly, the adaptive condition did not lead to significantly better learning results than the other two conditions on either the immediate or delayed posttest.

\section{Discussion and Conclusion}

Our results provide evidence that working with interactive erroneous examples can help students learn mathematics, delivering a learning experience similar to other types of desirable difficulties, one that facilitates deeper understanding over time instead of immediately.

However, the adaptive erroneous examples condition, which we hypothesized would be even better than the erroneous examples condition, did not result in higher learning gains. A blocked format of material presentation may have had a negative affect on the adaptive condition. In this condition many students displayed one (or two) prominent misconception(s) on the pretest and thus received a large number of problems of similar type(s), i.e., the blocking was very prominent in the adaptive condition. Thus, a modification to the adaptive algorithm to provide more problem variability (and thus more desirable difficulty) could make a big difference. 
Acknowledgements: The U.S. Department of Education (IES), Award No: R305A090460, provided support for this research. We also thank the Pittsburgh Science of Learning Center, NSF Grant \# 0354420, for technical support of our work.

\section{$5 \quad$ References}

1. Schmidt, R.A., Bjork, R.A.: New conceptualization of practice: Common principles in three paradigms suggest new concepts for training. Psych. Science, 3(4), 207-217 (1992)

2. Bjork, E. L., Bjork, R. A.: Making things hard on yourself, but in a good way: Creating desirable difficulties to enhance learning. In M. A. Gernsbacher, R. W. Pew, L. M. Hough, \& J. R. Pomerantz (Eds.), Psychology and the real world: Essays illustrating fundamental contributions to society (pp. 56-64). New York: Worth Publishers. (2011)

3. Irwin, K.C.: Using everyday knowledge of decimals to enhance understanding. Journal for Research in Mathematics Education, 32(4), 399-420 (2001)

4. Sackur-Grisvard, C., Léonard, F.: Intermediate cognitive organizations in the process of learning a mathematical concept: The order of positive decimal numbers. Cognition and Instruction, 2, 157-174 (1985)

5. Stacey, K., Helme, S., Steinle, V., Baturo, A., Irwin, K., Bana, J.: Preservice teachers' knowledge of difficulties in decimal numeration. Journal of Mathematics Teacher Education, 4, 205-225 (2001)

6. Renkl, A., Atkinson. R.K.: Learning from worked-out examples and problem solving. In. J.L. Plass, R. Moreno, \& R. Brünken (Eds.), Cognitive Load Theory, Cambridge: Cambridge University Press (2010)

7. Fonseca, B., Chi, M.T.H.: The self-explanation effect: A constructive learning activity. In Mayer, R. \& Alexander, P. (Eds.), The Handbook of Research on Learning and Instruction (pp. 270-321). New York, USA: Routledge Press. (2001)

8. Siegler, R.S.: Microgenetic studies of self-explanation. In N. Granott and J. Parziale (eds). Microdevelopment, Transition Processes in Development and Learning, (pp. 31-58). Cambridge University Press (2002)

9. Grosse, C.S., Renkl, A.: Finding and fixing errors in worked examples: Can this foster learning outcomes? Learning and Instruction, 17(6), 612-634 (2007)

10. Isotani, S., Adams, D., Mayer, R.E., Durkin, K., Rittle-Johnson, B., McLaren, B.M.: Can erroneous examples help middle-school students learn decimals? In: Proceedings of the Sixth European Conference on Technology Enhanced Learning: Towards Ubiquitous Learning (EC-TEL 2011) (pp. 181-195) (2011)

11. McLaren, B.M., Adams, D., Durkin, K., Goguadze, G. Mayer, R.E., Rittle-Johnson, B., Sosnovsky, S., Isotani, S., Van Velsen, M.: To err is human, to explain and correct is divine: A study of interactive erroneous examples with middle school math students. In: Proceedings of EC-TEL 2012: Seventh European Conference on Technology Enhanced Learning, LNCS 7563 (pp. 222-235) Springer, Berlin (2012) 